\title{
Characteristics of incidentally found thyroid nodules in computed tomography: comparison with thyroid scintigraphy
}

\author{
Shahin Zandieh ${ }^{*}$, Dina Muin ${ }^{1}$, Reinhard Bernt ${ }^{1}$, Karl Hittmair ${ }^{1}$, Joerg Haller ${ }^{1}$ and Klaus Hergan²
}

\begin{abstract}
Background: In our daily experience, the differentiation between a cold and hot nodule is a very important factor for further clinical management of the patient.

In this study, we compared the characteristics of incidentally found thyroid nodules detected on computed tomography (CT) to thyroid scintigraphy (TS).

Methods: Diagnostic reports from chest $C T$ with intravenous contrast and TS examinations performed from January 2013 to January 2016 were analyzed retrospectively. We identified 70 subjects: 50 with thyroid nodules and 20 control subjects without thyroid nodules. The examination time of the TS was a minimum of two to four months after a chest $C T$. Chest CTs were performed in the arterial phase after the application of contrast media.

Results: Patients with a cold nodule had a significantly lower Hounsfield Unit (HU) Nodule(N)/Parenchyma (P) ratio values than the patients with a hot or warm nodule $(P<0.05)$. The cut-off HU N/P ratio value with the highest sum of sensitivity and specificity for the prediction of a functioning nodule was 69 (95\% Cl: 0.79-0.95).

Conclusions: Our results imply that the HU N/P ratio of the thyroid nodule on the chest CT should be taken into account to assess the functionality of the nodule. A lower HU N/P ratio should alert the radiologist or nuclear medicine physician to the possibility that the nodule might be cold and thus more prone to malignancy.
\end{abstract}

Keywords: Computed tomography, Thyroid scintigraphy, Thyroid nodule, Incidentaloma, Technetium thyroid uptake

\section{Background}

Thyroid scintigraphy (TS) is a nuclear medicine method that generates a functional depiction of a thyroid based on the uptake degree of different radionuclides. TS as a diagnostic procedure helps the physician obtain information about the thyroid's functional state. In addition, it determines the size, shape, and position of the thyroid gland. TS can define the degree of function in a thyroid nodule that is palpable or found incidentally during a non-nuclear imaging procedure. Hot nodules are more often benign than cold lesions are. The most commonly used substance is radioactive technetium-99 m [1-4].

\footnotetext{
* Correspondence: zandieh.s@icloud.com

${ }^{1}$ Institute of Radiology and Nuclear Medicine, Hanusch Hospital Teaching

Hospital of Medical University of Vienna, Vienna, Austria

Full list of author information is available at the end of the article
}

After accumulation in the thyroid gland, gamma radiation is recorded by the gamma camera so that the thyroid is presented as an image and the function of the thyroid may be assessed. Simultaneously, the amount of radionuclide is measured in the thyroid gland, and the technetium thyroid uptake (TcTU) is determined.

The thyroid nodule is an anomalous growth of thyroid cells. Most of the thyroid nodules are benign; a small portion of thyroid nodules could inclose malignant cells. Most thyroid nodules cause no noticeable symptoms. Nodules in the thyroid are mostly found incidentally during routine physical examinations or during a diagnostic modality such as computed tomography $(\mathrm{CT})$ or sonography, which have to be done for entirely unrelated reasons. Thyroid nodules could cause hyperthyroidism due to increased production of thyroid hormones. However, most thyroid nodules are actually non-functioning. Thyroid nodules are an extremely common thyroid 
disorder. The frequency of these incidentalomas has ranged from as low as $2 \%$ to as high as $67 \%$ [5].

Thyroid incidentaloma is a non-symptomatic lesion that is detected in a diagnostic modality while evaluating for non-thyroid-related issues. In the majority of cases, they are found during ultrasound examinations of the neck, followed by $\mathrm{CT}$, magnetic resonance imaging (MRI) and 18 F-Fluor-Desoxyglucose positron emission tomography (18 F-FDG-PET).

A couple of studies have attempted to evaluate the diagnostic value of $\mathrm{CT}$ to differentiate malignant thyroid nodule lesions from benign thyroid nodules. In these studies, the histopathological results have been used as a reference standard [5]. Davis et al. reported a low possibility of malignancy in these thyroid nodules depending on other risk factors in their study. The histopathological results of most of these malignancies were small papillary carcinomas [6].

Knowing the type of nodule (i.e., cold, hot, or warm) is critical in the clinical routine. About $13 \%$ of cold nodules have the risk of malignancy [7]. In addition, identifying the characteristics of the nodule type is also important for further thyroid nodule management. Although the presence of a cold nodule could be due to benign conditions, such as cysts, macrocalcification, and less commonly, areas of fibrosis or changes after thyroiditis, a malign lesion cannot be excluded. A fine-needle biopsy is often necessary.

The prevalence of incidentally found nodules in the thyroid has been reported in a couple of mainly CT- and MRI-based studies. Yoon et al. [8] found incidental nodules in the thyroid nodules in $16 \%$ of participants in their study. They examined 734 patients who were not known to have thyroid disease using a 16-slice CT scan. They reported that $9 \%$ of the incidentally found thyroid nodules were malignant. $123 \mathrm{CT}$ scans and 108 MRI examinations of the neck were used in the study by Youserm et al. A prevalence of $16 \%$ for incidental thyroid nodules was reported by this study group [9].

In our daily experience, the differentiation between a cold and hot nodule is a very important factor for further clinical management of the patient. In this study, we compared the characteristics of incidental thyroid nodules detected on CT to TS. To our knowledge, this is the first study that conducts such a comparison.

\section{Methods}

\section{Ethical issues}

The local ethics committee granted ethical approval for this study. Informed consent was obtained from all participants. This study conformed to the Declaration of Helsinki.

\section{Patient selection}

We evaluated 70 subjects. In our study, we searched retrospective chest CT examinations performed from
January 2013 to January 2016 that contained the words "thyroid nodule" in the diagnostic report. In 50 reports where one thyroid nodule was diagnosed, the patients also had TS. We identified and included 50 patients (19 men, 31 women; age range: 51-90 years; mean age: 67 years) in our study. The mean body mass index (BMI) of the patients was 26, and the mean weight was $68 \mathrm{~kg}$. At the time of the CT and TS examinations, all patients were unmedicated. No treatment was performed between the two examinations. In addition, we added 20 control subjects (16 men, 4 women; age range: $52-77$ years; mean age: 64 years) without thyroid nodules in $\mathrm{CT}$ and Ultrasound examination. The mean BMI of the control subjects was 25, and the mean weight was $64 \mathrm{~kg}$. Patients with cysts, parenchymal changes after surgery, and radiodine therapy have been excluded. We selected only patients with one nodule detected incidentally on chest $\mathrm{CT}$ examination.

\section{CT examination protocol}

The CT scans were performed with a Brilliance 64-slice CT scanner (Philips Medical Systems Corporation, Cleveland, Ohio, USA). The following parameters for the 64-section multi-detector scanner were used: reconstructed slice thickness, $3 \mathrm{~mm}$; reconstruction increment, $2 \mathrm{~mm}$; $x$-ray tube current, $200 \mathrm{mAs}$; voltage, $120 \mathrm{kV}$; and collimation, $6.4 \times 0.63 \mathrm{~mm}$. A filtered back projection reconstruction technique was used.

The chest CT scans were done in the craniocaudal direction. The iodine contrast material dosage was 300 $\mathrm{mgI} / \mathrm{kg}$. The injection flow rate was $2.5 \mathrm{~mL} / \mathrm{s}$. The chest CT was performed during the arterial phase. Both arms were raised above the shoulder region. No artefacts were detected in the thyroid region on selected chest CT examinations. The average radiation dose associated with chest CT scanning was $7 \mathrm{mSV}$.

\section{Thyroid scintigraphy}

The thyroid scintigraphy in our study was done with Tc99-metastable pertechnetate. The scans were performed with a parallel high-resolution low-energy collimator at an energy setting of $140 \mathrm{KeV}$ photo peak for Tc- $99 \mathrm{~m}$. The scan was done $20 \mathrm{~min}$ after the intravenous administration of 75-90 MBq Tc-99 $\mathrm{m}$ pertechnetate. The examination time of the TS was a minimum of two to four months after a chest CT. Nodules are depicted as zones of increased (hot), identical uptake compared to the uptake of normal paranodular thyroid tissue (warm) or decreased (cold) tracer uptake.

\section{Thyroid ultrasound}

The US studies were performed with a Toshiba Aplio XG scanner (Toshiba Medical Systems, Tokyo, Japan) using an $18 \mathrm{MHz}$ convex probe. The examinations of 
the patients were performed in the supine position. To locate the thyroid and to evaluate the thyroid nodule echogenicity, the real-time B-mode ultrasonography was used. The echogenicity of the nodule was reported as isoechoic, hypoechoic, or hyperechoic in relation to the thyroid parenchymal glands. The echotexture was described as homogeneous or heterogeneous. The US examination was performed on the same day as the thyroid scintigraphy.

\section{Image analysis}

We have used our picture archiving and communication system (PACS) to fetch the CT images and transfer them to a standard viewer (Sectra; Version 15.2.4.1, Sweden). Two circular regions of interest (ROI) $(5 \mathrm{~mm})$ were placed in the center of the thyroid lesion, and another two ROI were placed in the thyroid normal parenchyma (5 mm) (Fig. 1). All chest CT studies were reviewed by a radiologist with 10 years of experience in chest imaging. We determined the HU density nodule to parenchyma ratio (HU N/P ratio) for two ROI in the thyroid nodule. We also determined the $\mathrm{HU}$ density nodule to control subject's parenchyma ratio ( $\mathrm{HU} \mathrm{N} / \mathrm{P}$-control ratio) for two ROI in the thyroid nodule, and another two ROI were placed in the thyroid normal parenchyma.

\section{Statistical analysis}

All data were recorded in a Microsoft Excel file, and the statistical analysis was conducted using SPSS (Version 22, IBM, USA) and ACOMED Statistic (Version 1, ACOMED, Germany) software. The results are presented as a box plot.

For quantitative analysis, we performed independent tests to estimate the differences in our results. To calculate the optimal cut-off value to maximize the sum of sensitivity and specificity and to estimate the area under the curve (AUC), receivers operating characteristic curve (ROC) analyses have been performed. Probability values of less than 0.05 were considered significant.

\section{Results}

The study included 50 patients with chest CT and a thyroid incidentaloma.

The mean thyroid nodule size was $21 \mathrm{~mm}$ (min 11, $\max 50$ ) on the CT of the chest in the axial plane. The hot nodules had a mean size of $22 \mathrm{~mm}$, and the cold nodules had a mean size of $24 \mathrm{~mm}$. The warm nodules had a mean size of $21 \mathrm{~mm}$ measured in the axial plane.

Hypofunctional nodules with diminished uptake (cold nodule) were seen in 20 patients (40\%) (Fig. 2). Twentythree patient $(46 \%)$ showed a moderate uptake (warm nodule) on a Tc-99 m-Thyroid scan. Excessive uptake of Tc-99 m-pertechnetate was seen in 7 patients $(14 \%)$ as defined by a hot nodule (Fig 3a, b).

On the ultrasound examination, the cold nodules were hypoechoic in 11 patients, isoechoic in 6 patients, and hyperechoic in 3 patients. The warm nodules were isoechoic in 12 patients, hypoechoic in 10 patients, and hyperechoic in 1 patient. Four of the patients with hot nodules were hypoechoic, and three were isoechoic.

The mean TSH value for patients with a cold nodule at the time of TS was $1.5 \pm 1$. Patients with a warm nodule had a mean TSH value of $2.2 \pm 2$ and with a hot nodule, a value of $0.8 \pm 1$.

Histopathalogy results of cold nodules from 16 patients revealed 2 patients with thyroid carcinoma, 1 with anaplastic carcinoma, and 1 with papillary carcinoma. In 2 cases inflammatory cells were present, and in $10 \mathrm{pa}$ tients the results were compatible with non-malignant adenoma. Two patients with a cold nodule refused surgery and FNA. Only 4 patients with hot or warm nodules had biopsies indicative of non-malignant adenoma.

Cold nodules had a median $56 \mathrm{HU}$ value on the chest CT. A median HU of 89 was detected for warm nodules on the chest CT. Hot nodules showed a median value of $86 \mathrm{HU}$ on the chest CT examination. Cold nodules had a mean $\mathrm{HU} \mathrm{N} / \mathrm{P}$ ratio value of $56 \pm 22$ on the chest $\mathrm{CT}$. A mean $\mathrm{HU} \mathrm{N} / \mathrm{P}$ ratio of $87 \pm 20$ was detected for warm

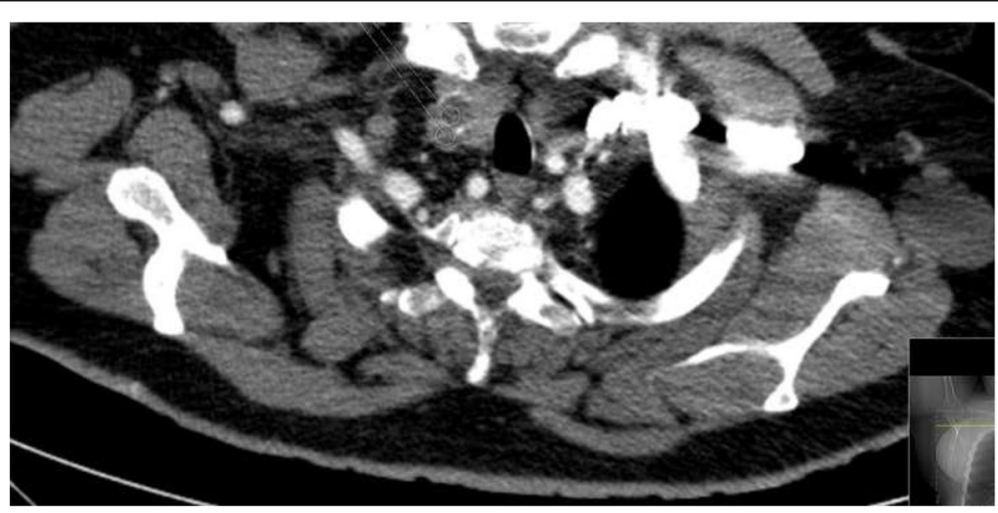

Fig. 1 The CT image shows two ROls in the thyroid nodule. Abbreviations: CT, computed tomography; ROI, region of interest 


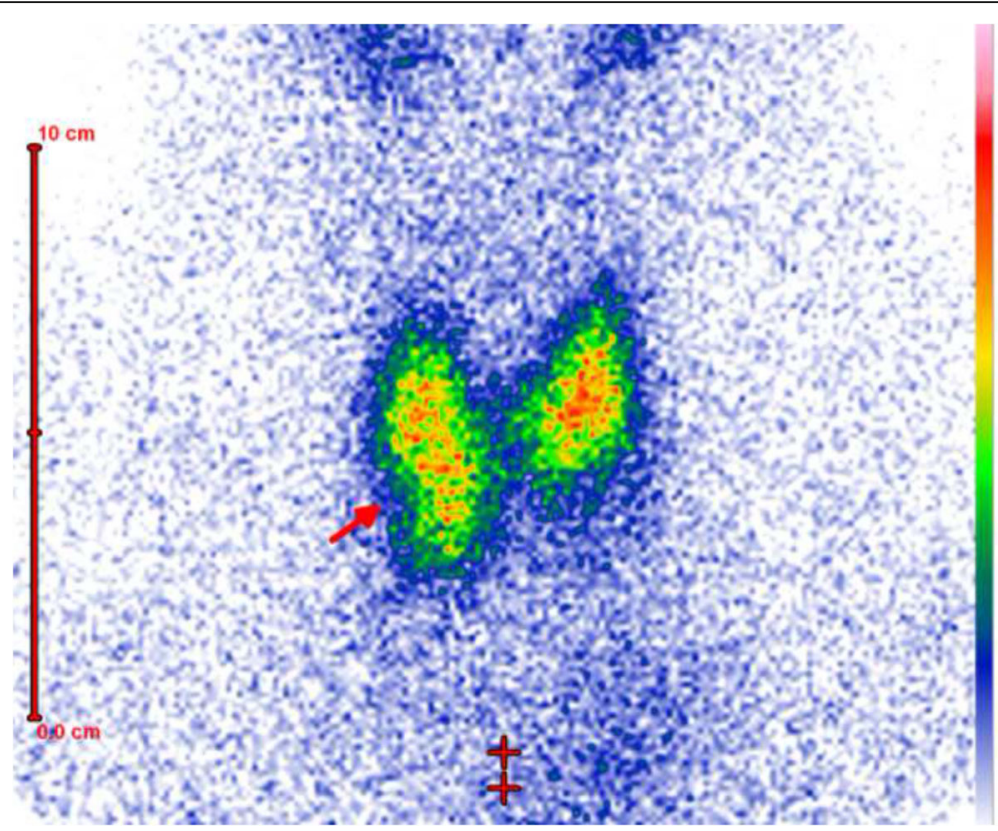

Fig. 2 TS shows an area with diminished uptake in the right lobe of the thyroid laterocaudal which is consistent with a cold nodule. Abbreviations: TS, Thyroid scintigraphy

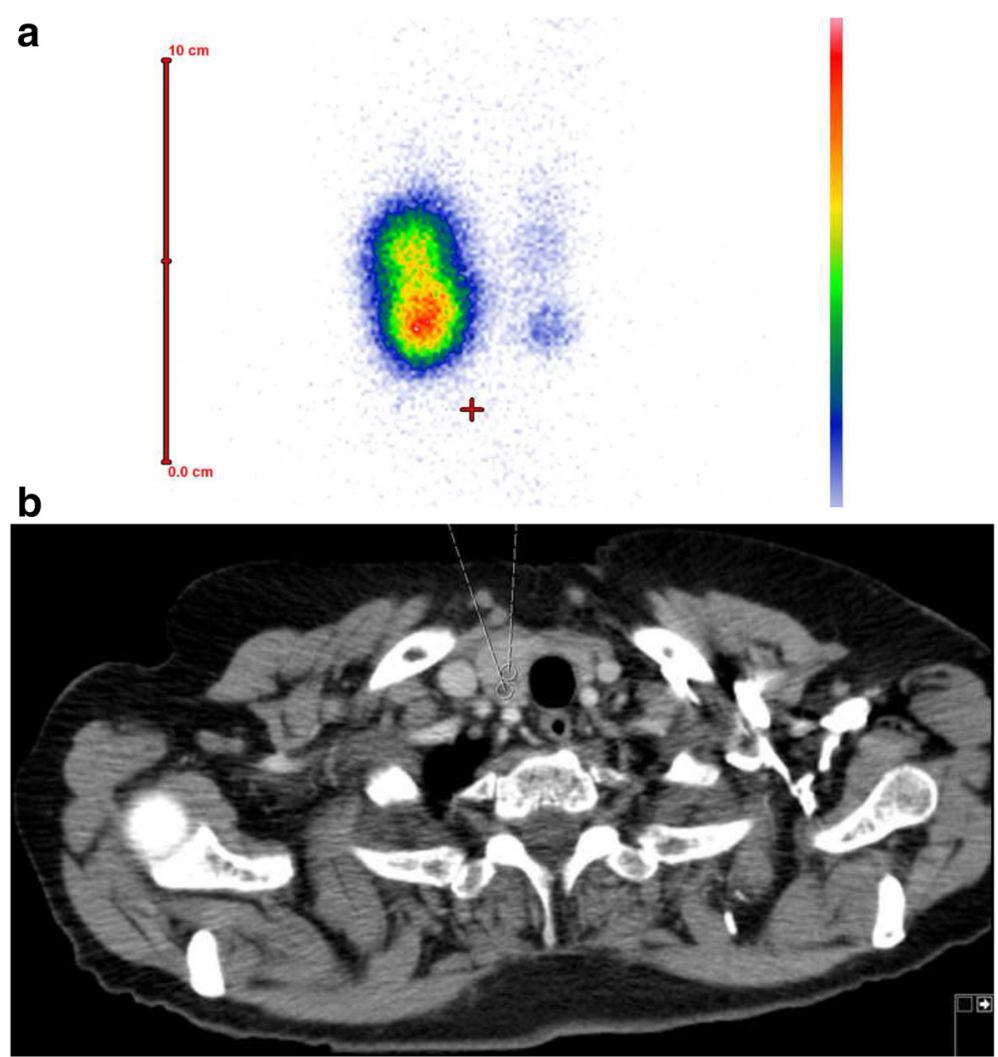

Fig. 3 a, b TS shows an area with increased uptake in the right lobe of the thyroid caudal which is consistent with a hot nodule The chest CT depicts a nodule in the right lobe of the thyroid. Abbreviations: TS, Thyroid scintigraphy; CT, computed tomography 
nodules on the chest CT. Hot nodules showed a mean value of $86 \pm 14 \mathrm{HU} \mathrm{N} / \mathrm{P}$ ratio on the chest CT examination. The HU N/P ratio values were significantly lower in patients with cold nodules than the other groups $(P<0.05)$ (Table 1$)$ (Fig. 4$)$.

Cold nodules had a mean HU N/P-control ratio value of $64 \pm 25$ on the chest CT. A mean HU N/P-control ratio of $94 \pm 21$ was detected for warm nodules on the chest CT. Hot nodules showed a mean value of $102 \pm 16$ HU N/P-control ratio on the chest CT examination. The HU N/P-control ratio values were significantly lower in patients with cold nodules than in the subjects of the other groups $(P<0.05)$.

The optimal cut-off HU N/P ratio value for which the sum of sensitivity and specificity was highest for the prediction of a functioning nodule (warm or hot) was 69 (95\% CI: 0.79-0.95). For this cut-off value, HU N/P ratio value had a sensitivity of $89 \%$ and a specificity of $83 \%$. To obtain a sensitivity $>90 \%$, the best cut-off value was 65 (95\% CI: 0.79-0.95) (94\% sensitivity, 78\% specificity) (Fig. 5) [10].

\section{Discussion}

Thyroid incidentaloma is an unintentional finding that is not related to the original reason for performing a CT study. In the last decade, the evolution of diagnostic techniques has significantly improved diagnoses in endocrine thyroid disease.

The challenge facing the clinician is to avoid unnecessary diagnostic intervention, treatment, or both [11]. Frank et al. reported in a systematic review of 11 chest CT studies that had been performed for coronary artery disease and lung cancer screening had revealed that the proportion of patients with at least one incidental imaging abnormality requiring follow-up varied from $3 \%$ to $41.5 \%$ [12].

Thyroid abnormalities, such as nodules, cysts, calcifications, and diffuse glandular enlargement, are often detected on chest CT examinations. Thyroid nodules are found in approximately $16 \%$ of chest CT scans, occurring more commonly in women than men. Most incidentally found thyroid lesions are benign, with an approximately $9 \%$ to $11 \%$ prevalence of malignant lesions [12].

Once an incidental thyroid nodule is found, referral to a thyroid specialist is necessary. According to American Thyroid Association (ATA) guidelines, generally, only nodules $>1 \mathrm{~cm}$ need further evaluation because they have a greater potential to represent clinically significant cancers compared with lesions $<1 \mathrm{~cm}$ and because of cost/benefit considerations regarding the work-up of small nodules.

According to Austrian clinical guidelines, after detection of a thyroid nodule of over $1 \mathrm{~cm}$, radionuclide imaging should be performed to characterize the thyroid nodule. If a nodule is cold, fine needle aspiration will be performed; otherwise, the nodule will be monitored on ultrasound besides there is a growth of the warm or hot nodule in the follow-up ultrasound examinations or FNA is recommended duo to the ATA guidelines. In radionuclide imaging, only $4 \%$ of hot nodules are known to contain malignant cells, compared with $16 \%$ of cold nodules [13].

Our results show no correlation between thyroid nodule functionality and US echogenicity. There was no correlation between the size of the thyroid nodule and functionality. The BMI values of the patient and the neck circumference measurements have no correlation to the thyroid nodule functionality.

Small amounts of free iodide are contained in an iodinated contrast medium. A 200-ml dose of a contrast medium containing $35 \mu \mathrm{g} / \mathrm{ml}$ provides 7,000 $\mu \mathrm{g}$ free iodide, equivalent to 45 times the recommended daily intake [14]. Our hypothesis was that iodine containing contrast media for chest $\mathrm{CT}$ accumulates in the thyroid due to the act of iodination, and this process could influence the HU values of the thyroid.

No CT feature is reliable in distinguishing cold from warm or hot thyroid lesions. In our study we tried to find a difference between the contrast uptakes of the thyroid nodule as detected incidentally by computed tomography and the degree of function of the same thyroid nodule on thyroid scintigraphy. Our data illustrate that there is a significant difference between the assessments, particularly, in the HU N/P ratio of the cold nodule to the hot or warm nodule. An HU N/P ratio values lower 69 should alert the radiologist of the presence of a cold nodule.

In addition, the results of the determination of the HU density nodule to control subject's parenchyma ratio in our study were significantly lower in patients with cold nodules than in the subjects of the other groups.

Table 1 Characteristics of CN, WN and HN detected incidentally on chest CT

\begin{tabular}{llllllr}
\hline & Patient & Mean size on CT & Isoechogenic & Hypoechogenic & Hyperechogenic & HU N/P ratio median \\
\hline CN & $20(40 \%)$ & $24 \mathrm{~mm}$ & 6 & 11 & 3 & $56 \pm 22$ \\
WN & $23(46 \%)$ & $21 \mathrm{~mm}$ & 12 & 10 & 1 & $87 \pm 20$ \\
$\mathrm{HN}$ & $7(14 \%)$ & $22 \mathrm{~mm}$ & 3 & 4 & 0 & $86 \pm 14$ \\
\hline
\end{tabular}

Abbreviations: $C N$ cold nodule; $W N$ warm nodule; $H N$ hot nodule; $C T$ computed tomography 


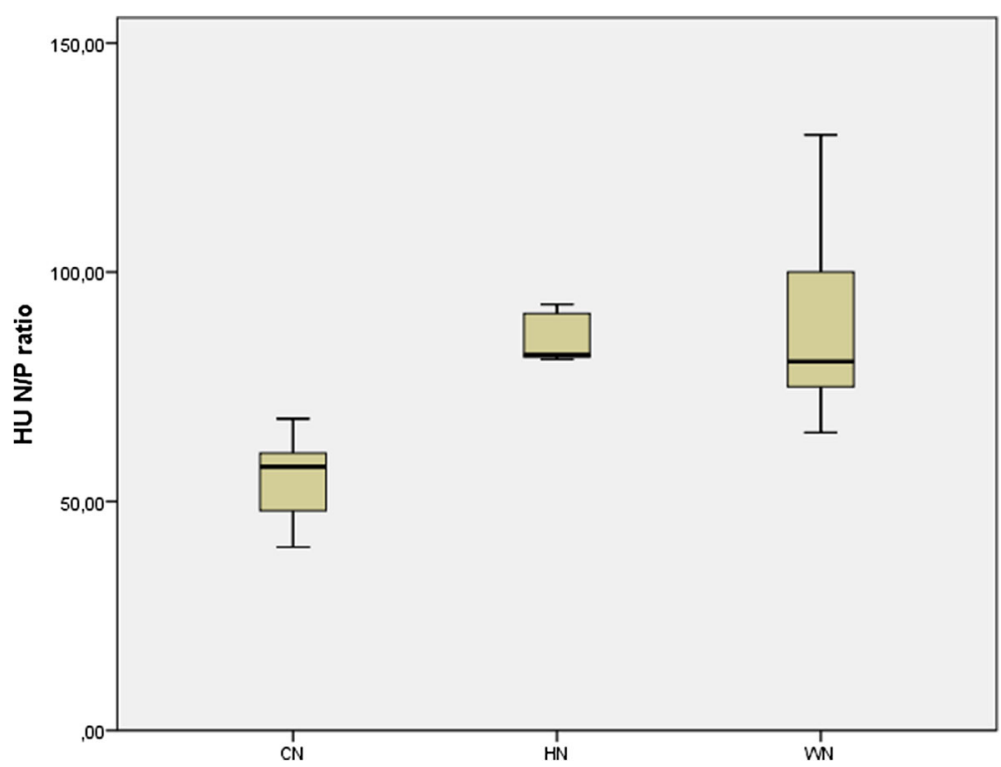

Fig. 4 Boxplot graphics of $H U N / P$ ratio values of $C N$, WN and HN. The HU N/P ratio values were lower in $C N$ than to $W N$ and $H N(P<0.05)$. Abbreviations: HU N/P ratio, Hounsfield Unit Nodule/Parenchyma ratio; CN, cold nodule; WN, warm nodule; HN, hot nodule

Patients who have been referred to a thyroid specialist to diagnose the thyroid nodule could have had a prior chest CT for other reasons.

ATA and ETA (European Thyroid Association) guidelines suggest that nodules that are "hot or warm on scintigraphy" do not require a biopsy.

Our results suggest that the HU N/P ratio of the thyroid nodule on a chest $\mathrm{CT}$ should be taken into account

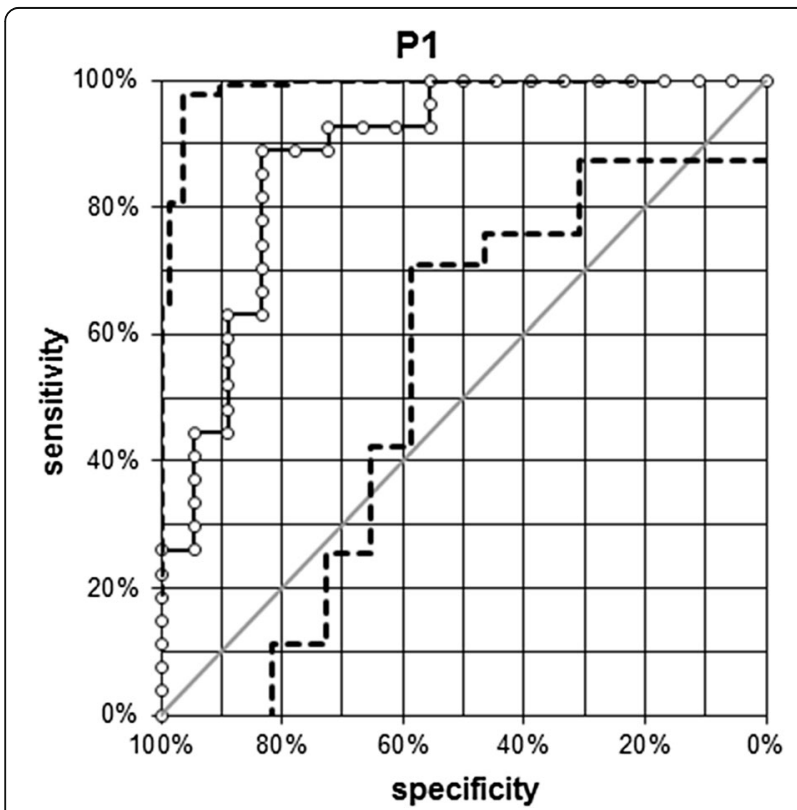

Fig. 5 The ROC curve of the HU N/P ratio values assessed in patients with thyroid nodule. Abbreviations: ROC, Receiver Operating Characteristic; HU N/P ratio, Hounsfield Unit Nodule/Parenchyma ratio to assess the functionality of the nodule before using thyroid scintigraphy. With further studies in the future, it may not be necessary to perform a TS in this context, which would avoid unnecessary radiation exposure and reduce costs.

In addition to the ultrasound characteristic of the nodule, a lower HU N/P ratio should alert thyroid specialists to the possibility that the nodule might be cold and thus more prone to malignancy, even using ATA and ETA guidelines for handling incidentalomas. In such cases, the biopsy could be done earlier.

In the study of Basharat et al., where a comparison was made between the results of TS and histopathology, the overall sensitivity on the TS was found to be $80 \%$, specificity was $20 \%$, positive predictive value was $10 \%$, and negative predictive value was $90 \%$. The overall accuracy was $26 \%$ [15]. In one study of TS, it was reported that the sensitivity was $100 \%$ and that the specificity was $24 \%$ [16].

False-positive TS is rare. Some artifacts and physiologic variants are known to consistently take up Tc-99metastable pertechnetate, but these are easily discounted by experienced nuclear medicine physicians $[17,18]$.

Limitations of the study included a lack of pathological results and differentiation between benign and malignant thyroid nodules; however, these were not relevant to the aim of the study. Another limitation is calcification. This should not be captured in the ROI in order to avoid sustaining inadequate $\mathrm{HU} \mathrm{N} / \mathrm{P}$ ratio values. In our study, both arms were raised above the shoulder region. We have not included data about other arm positions. Therefore, further studies are necessary to determine the optimal cut-off HU N/P ratio for other arm positions. 
We believe that with further studies, the guidelines could be reconsidered in the future.

\section{Conclusions}

Our results imply that the HU N/P ratio of the thyroid nodule on the chest $\mathrm{CT}$ should be taken into account to assess the functionality of the nodule. A lower HU N/P ratio should alert the radiologist or nuclear medicine physician to the possibility that the nodule might be cold and thus more prone to malignancy, even using ATA and ETA guidelines for handling incidentalomas.

\section{Abbreviations}

18 F-FDG-PET: 18 F-Fluor-Desoxyglucose positron emission tomography; ATA: American Thyroid Association; AUC: Area under the curve; CT: Computed tomography; ETA: European Thyroid Association; HU: Hounsfield Unit; MRI: Magnetic resonance imaging; PACS: Picture archiving and communication system; ROC: Receivers operating characteristic curve; ROI: Regions of interest; TCTU: Technetium thyroid uptake; TS: Thyroid scintigraphy

\section{Acknowledgements}

Not applicable.

\section{Funding}

The study was not funded.

\section{Availability of data and materials}

Data to replicate findings are presented in the Tables of the main paper. Due to patient privacy protection, any additional materials of the study are only available upon individual request directed to the corresponding author.

\section{Authors' contributions}

SZ conceived the study and design. SZ, DM and RB undertook acquisition of data. SZ and RB analyzed and interpreted the data and drafted the manuscript. $\mathrm{JH}, \mathrm{KHM}$ performed critical revision of the manuscript. KH supervised the study. All authors read and approved the final manuscript.

\section{Competing interest}

The authors declare that they have no competing interests.

\section{Consent for publication}

Not applicable.

\section{Ethics approval and consent to participate}

The local ethics committee of Vienna granted ethical approval for this study. Informed consent was obtained from all participants. This study conformed to the Declaration of Helsinki.

\section{Author details}

${ }^{1}$ Institute of Radiology and Nuclear Medicine, Hanusch Hospital Teaching Hospital of Medical University of Vienna, Vienna, Austria. ${ }^{2}$ Department of Radiology, Paracelsus Medical University of Salzburg, Salzburg, Austria.

Received: 3 October 2016 Accepted: 9 January 2017

Published online: 21 January 2017

\section{References}

1. Burke G, Halko A, Silverstein GE, et al. Comparative thyroid uptake studies with 131I and 99mTcO4. J Clin Endocrinol Metab. 1972;34:630-7.

2. Ramos CD, Zantut Wittmann DE, Tambascia MA, et al. Thyroid suppression test with L-thyroxine and [99mTc] pertechnetate. Clin Endocrinol. 2000;52:471-7.

3. Andros G, Harper PV, Lathrop KA, et al. Pertechnetate- 99 m localization in man with applications to thyroid scanning and the study of thyroid physiology. J Clin Endocrinol Metab. 1965;25:1067-76.

4. Smith JJ, Croft BY, Brookeman VA, et al. Estimation of 24-h thyroid uptake of -131 sodium iodide using a 5-min uptake of technetium-99 m pertechnetate. Clin Nucl Med. 1990;15:80-3.
5. Ahmed S, Horton KM, Jeffrey Jr RB, et al. Incidental thyroid nodules on chest CT: Review of the literature and management suggestions. AJR Am J Roentgenol. 2010;195(5):1066-71.

6. Davies L, Welch HG. Increasing incidence of thyroid cancer in the United States, 1973-2002. JAMA. 2006;295(18):2164-7.

7. McCall A, Jarosz H, Lawrence AM, et al. The incidence of thyroid carcinoma in solitary cold nodules and in multinodular goiters. Surgery. 1986;100(6): 1128-32. 295:2164-2167.

8. Yoon DY, Chang SK, Choi CS, et al. The prevalence and significance of incidental thyroid nodules identified on computed tomography. J Comput Assist Tomogr. 2008;32:810-5.

9. Youserm DM, Huang T, Loevner LA, et al. Clinical and economic impact of incidental thyroid lesions found with CT and MR. AJNR. 1997;18:1423-8.

10. Hanley JA, Mcneil BJ. The meaning and use of the area under a receiver operating characteristic (ROC) curve. Radiology. 1982;143(1):29-36.

11. Burguera B, Gharib H. Thyroid incidentaloma. Endocrinol Metab Clin North Am. 2000;29(1):187-203.

12. Frank L, Quint LE. Chest CT incidentalomas: thyroid lesions, enlarged mediastinal lymph nodes, and lung nodules. Cancer Imaging. 2012;12:41-8.

13. Daumerie C, Ayoubi S, Rahier J, et al. Prevalence of thyroid cancer in hot nodules. Ann Chir. 1998;52(5):444-8.

14. Van der Molen A, Thomsen H, Morcos S. Effect of iodinated contrast media on thyroid function in adults. Eur Radiol. 2004;14(5):902-7.

15. Basharat R, Bukhari MH, Saeed S, Hamid T. Comparison of fine needle aspiration cytology and thyroid scan in solitary thyroid nodule. Pathol Res Int. 2011:2011:754041.

16. Bapat RD, Pai P, Shah S, Bhandarkar SD. Surgery for thyroid goiter in western India. A prospective analysis of 334 cases. J Postgrad Med. 1993;39(4):202-4.

17. Smith EM. Properties, uses, radiochemical purity and calibration of Tc99m. Jucl Med. 1964;5:871-82.

18. Lebo NL, Raymond F, Odell MJ. Selectively false-positive radionuclide scan in a patient with sarcoidosis and papillary thyroid cancer: a case report and review of the literature. J Otolaryngol Head Neck Surg. 2015;44(1):18.
Submit your next manuscript to BioMed Central and we will help you at every step:

- We accept pre-submission inquiries

- Our selector tool helps you to find the most relevant journal

- We provide round the clock customer support

- Convenient online submission

- Thorough peer review

- Inclusion in PubMed and all major indexing services

- Maximum visibility for your research

Submit your manuscript at www.biomedcentral.com/submit
Biomed Central 